\title{
The role of the TetR-family transcriptional regulator Aur1R in negative regulation of the auricin gene cluster in Streptomyces aureofaciens CCM 3239
}

Correspondence

Jan Kormanec

jan.kormanec@savba.sk

Received 4 January 2010

Revised 5 May 2010

Accepted 6 May 2010

\author{
Renata Novakova, Peter Kutas, Lubomira Feckova and Jan Kormanec \\ Institute of Molecular Biology, Slovak Academy of Sciences, 84551 Bratislava, Slovak Republic
}

\section{INTRODUCTON}

Gram-positive, filamentous, soil-dwelling bacteria of the genus Streptomyces are characterized by a complex morphological differentiation and by the ability to produce an extraordinary diversity of biologically active secondary metabolites, including the majority of known antibiotics. Antibiotic production is co-ordinated with morphological differentiation and often occurs in a growth phasedependent manner, usually at the beginning of the stationary growth phase, and is also influenced by a variety of physiological and environmental factors. Most antibiotics are produced by complex biosynthetic pathways encoded by physically clustered genes. The gene clusters are usually regulated by pathway-specific transcriptional activators of different families that are located in these clusters and act as a master switch for biosynthesis of the respective secondary metabolite. In addition to the transcriptional activators, expression of the antibiotic biosynthetic genes is often negatively regulated by transcriptional regulators that belong mainly to the TetR family. Moreover, various global regulatory genes affect antibiotic production indirectly and

Abbreviations: EMSA, electrophoretic mobility-shift assay; PKS, polyketide synthase; TSP, transcription start point. have pleiotropic roles in stress response and morphological differentiation. Many of these pleiotropic regulatory genes influence the activity of the pathway-specific regulatory genes (Bibb, 2005).

We have previously identified a type II polyketide synthase (PKS) gene cluster, aurl, which is responsible for production of the angucycline-like polyketide antibiotic auricin in Streptomyces aureofaciens CCM 3239 (Novakova et al., 2002). Even though auricin has been clearly detected in S. aureofaciens CCM 3239 grown in Bennet medium (Novakova et al., 2002), its purification and complete structural elucidation, which are currently under investigation, are hampered by its extremely low productivity (J. Kormanec, unpublished results). Similar problems have been recently described for several other homologous angucycline clusters (Lombo et al., 2004; Metsa-Ketela et al., 2004; Pang et al., 2004). This indicates that these types of PKS clusters are very tightly regulated. Knowledge of the mechanisms of their regulation at the molecular level is therefore of great interest, and would potentially allow an increase in the yields of auricin in the producer strain.

Two putative regulatory genes, aurlP and aurlR, whose deduced products share significant similarity with two 
different types of bacterial regulatory proteins, have been previously identified upstream of the aurl polyketide gene cluster in S. aureofaciens CCM 3239. The aurlP gene, encoding a protein similar to response regulators of bacterial two-component signal transduction systems, has been characterized in more detail. It has been shown to specifically activate expression of the auricin biosynthetic genes (Novakova et al., 2005). The divergent aur1R gene has been shown to encode a protein homologous to transcriptional repressors of the TetR family. Members of this family function mainly as repressors and regulate genes encoding biosynthetic enzymes for antibiotics, drug-efflux pumps and other proteins. The structure of the TetRfamily proteins consists of two domains: a DNA-binding domain which contains a helix-turn-helix motif and a regulatory domain that recognizes the signals via ligand binding. TetR-family proteins show high sequence similarity in their DNA-binding domains and almost no significant similarity in the regulatory domains (Ramos et al., 2005).

The aim of the present study was to characterize the aur $1 R$ gene in S. aureofaciens CCM 3239. For this purpose the gene was inactivated by gene replacement and auricin production was investigated in the mutant. Gene expression analysis, involving S1 nuclease mapping, was applied to the known aurl promoters in the aurlR mutant as well as in the wild-type strain. We further showed that Aur1R directly binds one of the promoters, aurlPp, and that the binding is affected by auricin or its intermediates. The results presented here show that Aur1R is a transcriptional repressor of the aur1P gene, which encodes a pathwayspecific activator of the auricin biosynthetic gene cluster in S. aureofaciens CCM 3239.

\section{METHODS}

Bacterial strains, plasmids and culture conditions. S. aureofaciens CCM 3239 wild-type was from the Czechoslovak Collection of Micro-organisms (Brno, Czech Republic). Escherichia coli cosmid SuperCos-1 (Evans et al., 1989) was from Stratagene. E. coli BW25113/pIJ790 was used as a host for PCR-targeted gene disruption using the apramycin-resistance plasmid pIJ773, and E. coli ET12567/ pUZ8002 was used as a non-methylating host (Gust et al., 2003). The Streptomyces integrative plasmid pPM927 (Smokvina et al., 1990) was provided by Mark J. Buttner (John Innes Institute, Norwich, UK). The plasmid pJUP3, encompassing the aur $1 R$ and aur $1 P$ region, has been described previously (Novakova et al., 2005). E. coli BL21(DE3) pLysS and the plasmid pET28a, used for aur1R overexpression, were obtained from Novagen. Growth and transformation of S. aureofaciens CCM 3239 strains were carried out as described in Kormanec et al. (1993). The phenotype of the S. aureofaciens $\Delta a u r 1 R$ mutant was analysed after growth on solid minimal MM medium (Kieser et al., 2000) and rich Bennet medium (Horinouchi et al., 1983), and after growth in liquid minimal NMP medium (Kieser et al., 2000) and rich Bennet medium. For RNA isolation, $5 \times 10^{8}$ c.f.u. of the particular $S$. aureofaciens CCM 3239 strain were inoculated in $50 \mathrm{ml}$ liquid Bennet medium and grown at $28{ }^{\circ} \mathrm{C}$ to different growth phases. Conditions for E. coli growth and transformation have been described by Ausubel et al. (1995).
DNA manipulations. The standard DNA manipulations in E. coli were done as described in Ausubel et al. (1995), and those in Streptomyces according to Kieser et al. (2000). Chromosomal DNA from S. aureofaciens CCM 3239 strains was isolated according to Kieser et al. (2000). A cosmid library of partially Sau3AI-digested S. aureofaciens CCM 3239 chromosomal DNA fragments cloned in the BamHI site of SuperCos-1 was constructed essentially as described in Redenbach et al. (1996). The cosmid library (5000 clones) was screened with a 670 bp SacI DNA fragment comprising the aurlMaur $1 N$ genes from the end of the previously cloned $15.1 \mathrm{~kb}$ region of the aurl cluster (Novakova et al., 2002). A total of 28 positive clones were identified, and the corresponding recombinant cosmids pCosSA1-28 isolated and analysed by restriction mapping and hybridization. One cosmid, pCosSA25, containing the largest region upstream of the aurlR gene in the aurl cluster, has been used in further studies for aurlR disruption.

Disruption of the S. aureofaciens CCM 3239 aur1R gene. The PCR-targeted REDIRECT procedure (Gust et al., 2003) was used to delete the aurlR gene in S. aureofaciens CCM 3239. In brief, the apramycin-resistance cassette was PCR-amplified using primers Aur1Rdir (5' -TTTAATAATAGAAGGAAACTGATACAGGAGCTATGCGTGATTCCGGGGATCCGTCGACC-3') and Aur1Rrev (5' -GGCTGCCAGACAGGGCATGACCACCCGCCAGAACCCCGCTGTAGGCTGGAGCTGCTTC-3') and the template plasmid pIJ773. The resulting PCR product was introduced by electroporation into $E$. coli BW25113/pIJ790 containing cosmid pCosSA25. The correct replacement of the aurlR-coding region in the resulting SuperCos1-derived cosmid pCosSA25::AmR was verified by restriction mapping. The cosmid pCosSA25::AmR was transformed into the non-methylating E. coli ET12567/pUZ8002 strain and introduced into S. aureofaciens CCM 3239 by conjugation, and mutants resistant to apramycin and sensitive to kanamycin were selected. Two apramycin-resistant and kanamycin-sensitive colonies were identified, and for each the correct double-crossover event was proved by Southern blot hybridization (data not shown). Both clones had a similar phenotype, and the first strain, S. aureofaciens $\Delta a u r 1 R 1$, was chosen for further study. The plasmid pPM-aur1R, used for complementation of the aur1R mutation, was prepared by inserting a $1.25 \mathrm{~kb} B a m \mathrm{HI}-\mathrm{NcoI}$ (bluntended) fragment of plasmid pJUP3 (containing the aur1R gene with its promoter) in pPM927 cut with BamHI and KpnI (blunt-ended).

RNA isolation and S1 nuclease mapping. Isolation of total RNA from S. aureofaciens CCM 3239 and high-resolution S1 nuclease mapping were performed according to Kormanec (2001). Samples $(40 \mu \mathrm{g})$ of RNA were hybridized to approximately $0.015 \mathrm{pmol}$ of suitable DNA probe labelled at one $5^{\prime}$ end with $\left[\gamma^{32} \mathrm{P}\right] \mathrm{ATP}$ $\left[\sim 10^{6}\right.$ d.p.m. pmol ${ }^{-1}\left(60 \mathrm{MBq} \mathrm{pmol}^{-1}\right)$ of probe]. The $\mathrm{S} 1$ probes were prepared as follows. Probe 1 (for the aurlRp promoter) was prepared by PCR amplification from plasmid pJUP3 using the $5^{\prime}$ endlabelled primer Aur1RS1 (5'-GCTCAGCGTCGCCTCTGCGTACC$3^{\prime}$ ) from the aur1R-coding region and the unlabelled primer Aur1PS1 (5'-CCTTCGGCCGGTGAGAAACC-3') from the aur $1 P$-coding region. Probe 2 (for the aurlPp promoter) was prepared similarly by PCR amplification using $5^{\prime}$ end-labelled primer Aur1PS1 and unlabelled primer Aur1RS1. Probe 3 (for the aur1Ap promoter) has been described previously (Novakova et al., 2005), and the control hrdBp2 promoter probe has been described in Kormanec \& Farkasovsky (1993). The primers were labelled at the $5^{\prime}$ end with $\left[\gamma^{-32} \mathrm{P}\right] \mathrm{ATP}[\mathrm{ICN}, 4500 \mathrm{Ci}$ $\mathrm{mmol}^{-1}\left(166.5 \mathrm{TBq} \mathrm{mmol}^{-1}\right)$ ] and $\mathrm{T} 4$ polynucleotide kinase, as described by Ausubel et al. (1995). The protected DNA fragments were analysed on DNA sequencing gels together with $\mathrm{G}+\mathrm{A}$ and $\mathrm{T}+\mathrm{C}$ sequencing ladders derived from the end-labelled fragments (Maxam \& Gilbert, 1980).

Overexpression of aur1R in E. coli and protein purification. The aur $1 R$ gene was amplified by PCR using pJUP3 as a template and the 
primers aur1RNde (5'-GATACAGGAGCTACATATGATCAAACAAG- $3^{\prime}$ ) and aur1RHind (5'-GGGGAAGCTTCAGGAGCCGCGGCCCGCAGCGC- $3^{\prime}$ ), which introduced an NdeI site (in bold type) overlapping the translation initiation codon and a HindIII site (in bold type) downstream of the stop codon, respectively. To ensure higher fidelity of DNA synthesis during PCR, $P f u$ DNA polymerase (Stratagene) was used. A $620 \mathrm{bp}$ aurlR-containing DNA fragment was cut with NdeI and HindIII and cloned in pET28a cut with the same enzymes, resulting in pET-aur1R, which was verified by nucleotide sequencing. The host strain for pET-series expression plasmids, E. coli BL21 (DE3) pLysS, transformed with the plasmid pET-aur1R, was grown in LB medium containing $30 \mu \mathrm{g}$ chloramphenicol ml $\mathrm{m}^{-1}$ and $40 \mu \mathrm{g}$ kanamycin $\mathrm{ml}^{-1}$ at $30{ }^{\circ} \mathrm{C}$ to $\mathrm{OD}_{600} 0.5$. Expression was induced with $1 \mathrm{mM}$ IPTG. After $3 \mathrm{~h}$, the cells were harvested by centrifugation at $12000 \mathrm{~g}$ for $10 \mathrm{~min}$ and washed with ice-cold $0.9 \%(\mathrm{w} / \mathrm{v}) \mathrm{NaCl}$. The lysis of cells and native purification of His-tagged Aur1R protein on His-Tag Bind resin (Novagen) were carried out as directed by the manufacturer. The eluted proteins were dialysed overnight at $4{ }^{\circ} \mathrm{C}$ against the storage buffer [12.5 mM Tris/ $\mathrm{HCl}, \mathrm{pH} 7.9,60 \mathrm{mM} \mathrm{KCl}, 1 \mathrm{mM}$ EDTA, $1 \mathrm{mM} \mathrm{DTT}, 50 \%(\mathrm{v} / \mathrm{v})$ glycerol] and cleared by centrifugation at $30000 \mathrm{~g}$ for $10 \mathrm{~min}$, and the supernatant was stored in aliquots at $-20{ }^{\circ} \mathrm{C}$. The overproduction and purity of the eluted protein were confirmed by SDS-PAGE (Laemmli, 1970). A prominent band $\left(M_{\mathrm{r}} 24500\right)$ was identified after IPTG induction and isolation by His-Tag Bind resin (data not shown). This value corresponded to the calculated $M_{\mathrm{r}}$ of the $6 \times$ Histagged Aur1R protein (24012). The concentration of protein was determined according to Bradford (1976) with BSA as a standard.

Analysis of secondary metabolites. Spores $\left(5 \times 10^{8}\right.$ c.f.u $)$ of $S$. aureofaciens CCM 3239 and the corresponding S. aureofaciens $\Delta$ aurlR1 mutant strains were inoculated into $50 \mathrm{ml}$ Bennet medium in $250 \mathrm{ml}$ Erlenmayer flasks, and the cultures were grown on an orbital shaker at 270 r.p.m. and $28{ }^{\circ} \mathrm{C}$. Aliquots $(4 \mathrm{ml})$ were taken from the cultures at particular time points and pelleted by centrifugation $\left[8000 \mathrm{~m} \mathrm{~s}^{-1}\right.$ $(820 \mathrm{~g}), 5 \mathrm{~min}]$. Supernatants were extracted twice with the same volume of ethyl acetate. Residual water was removed with sodium sulphate and sodium chloride, and extracts were evaporated under vacuum. The pellets were dissolved in $30 \mu \mathrm{lmethanol,} \mathrm{and} 5 \mu \mathrm{l}$ aliquots were subjected to TLC analysis on silica gel $60 \mathrm{~F}_{254}$ plates (Merck) with n-butanol saturated with water. Dried TLC plates were analysed by biochromatography by placing them on LB agar plates and overlaying with soft nutrient agar (Kieser et al., 2000) containing a fresh culture of Bacillus subtilis. The agar was allowed to solidify and plates were incubated for $16 \mathrm{~h}$ at $37{ }^{\circ} \mathrm{C}$ and screened visually for growth-inhibition zones. Extracts used for electrophoretic mobility shift assays (EMSAs) were prepared from S. aureofaciens CCM 3239 and S. aureofaciens, aurl:: tsr (Novakova et al. 2002) grown in $50 \mathrm{ml}$ of medium as described above, although the whole culture medium was extracted twice with ethyl acetate and finally dissolved in $30 \mu \mathrm{l}$ methanol.

EMSA. A 200 bp SmaI-EcoRI DNA fragment encompassing the aurlAp promoter uniquely labelled on the $5^{\prime}$ end at the EcoRI site has been described previously (Novakova et al., 2005). A 406 bp fragment (probe 2) comprising the aurlR-aurlP intergenic region [nucleotide positions -291 to +115 with respect to the transcription start point (TSP) for the aurlPp promoter] was prepared by PCR amplification from pJUP3 using the $5^{\prime}$ end-labelled primer Aur1PS1 and unlabelled primer Aur1RS1. EMSA was done essentially as described by Ausubel et al. (1995). The ${ }^{32} \mathrm{P}$-labelled DNA fragments [0.04 ng, 2000 d.p.m. $(120 \mathrm{kBq})]$ were incubated with increasing amounts of purified Aur1R protein for $15 \mathrm{~min}$ at $30{ }^{\circ} \mathrm{C}$ in a $15 \mu \mathrm{l}$ total volume of the binding buffer $[12.5 \mathrm{mM}$ Tris, $\mathrm{pH} 7.9,60 \mathrm{mM} \mathrm{KCl}, 1 \mathrm{mM}$ EDTA, $1 \mathrm{mM}$ DTT, $12 \%(\mathrm{v} / \mathrm{v})$ glycerol], $2 \mu \mathrm{g}$ sonicated salmon sperm DNA and $4.5 \mu \mathrm{g}$ BSA. After incubation, protein-bound and free DNA was resolved on a non-denaturing polyacrylamide gel containing $4 \%(\mathrm{w} / \mathrm{v})$ acrylamide, $0.05 \%(\mathrm{w} / \mathrm{v})$ bisacrylamide and $2.5 \%(\mathrm{w} / \mathrm{v})$ glycerol, running at $4{ }^{\circ} \mathrm{C}$ (after a $1 \mathrm{~h}$ prerun at $30 \mathrm{~mA}$ ) in a high-ionic-strength buffer containing $50 \mathrm{mM}$ Tris, $380 \mathrm{mM}$ glycine, 2 mM EDTA, pH 8.5, at the same current. The gels were dried and exposed to an X-ray film.

The effect of auricin on the ability of AurlR to bind the DNA probe was tested by adding $1 \mu \mathrm{l}$ of the undiluted extracts or a series of twofold-diluted extracts (with methanol) to the $15 \mu$ EMSA binding reaction.

DNase I footprinting. Binding reactions were performed in $30 \mu \mathrm{l}$ binding buffer at the same conditions as for the EMSA with $0.3 \mathrm{ng}$ [ 12000 d.p.m. (720 kBq)] of either the coding or non-coding strand 5 ' end-labelled 406 bp DNA fragment encompassing the aur1RaurlP intergenic region (probe 1 and probe 2, respectively) and increasing amounts of purified Aur1R. After incubation for $15 \mathrm{~min}$ at $30{ }^{\circ} \mathrm{C}, 3 \mu \mathrm{l}$ DNase I solution [5 U DNase I ml ${ }^{-1}$ (Roche) in $100 \mathrm{mM}$ $\mathrm{MgCl}_{2}, 100 \mathrm{mM}$ DTT] was added to the binding reaction. The reaction was incubated for $40 \mathrm{~s}$ at $37{ }^{\circ} \mathrm{C}$, stopped by adding $7.5 \mu \mathrm{l}$ DNase I stop buffer ( $3 \mathrm{M}$ ammonium acetate, $0.25 \mathrm{M}$ EDTA, $0.1 \mathrm{mg}$ tRNA $\mathrm{ml}^{-1}$ ) and extracted with $30 \mu \mathrm{l}$ alkaline phenol/chloroform. The aqueous phase was precipitated by adding three volumes of ethanol. The resulting pellet, after washing with $70 \%(\mathrm{v} / \mathrm{v})$ ethanol and SpeedVac drying, was suspended in $5 \mu \mathrm{l}$ Maxam loading buffer [80\% (v/v) formamide, $1 \mathrm{mM}$ EDTA, $10 \mathrm{mM} \mathrm{NaOH}, 0.05 \%$ (w/v) bromophenol blue, $0.05 \%(\mathrm{w} / \mathrm{v})$ xylene cyanol $\mathrm{FF}$. The DNA fragments were analysed on $6 \%$ DNA sequencing gels together with $\mathrm{G}+\mathrm{A}$ and $\mathrm{T}+\mathrm{C}$ sequencing ladders derived from the end-labelled fragment (Maxam \& Gilbert, 1980). After electrophoresis, the gels were dried and exposed to an X-ray film.

\section{RESULTS}

\section{Disruption of the aur1R gene positively affects auricin production}

Expression of the auricin biosynthetic genes in S. aureofaciens CCM 3239 has been previously shown to be under the control of the pathway-specific positive regulator Aur1P, which belongs to the family of response regulators of bacterial two-component signal transduction systems. A divergently transcribed gene, aur $1 R$, has been identified upstream of aurlP and shown to encode a repressor belonging to the TetR family (Novakova et al., 2005). To investigate the role of the aur $1 R$ gene in auricin biosynthesis and regulation, a chromosomal copy of $S$. aureofaciens aurlR was inactivated using a PCR targeting system (REDIRECT method) for disruption of Streptomyces genes (Gust et al., 2003). The mutation did not affect growth and differentiation (data not shown), and the mutant strain $S$. aureofaciens $\triangle$ aur $1 R 1$ grew at a similar rate to the wild-type strain (Fig. 1a). Wild-type S. aureofaciens CCM 3239 and the S. aureofaciens $\Delta$ aur1R1 mutant were investigated for production of auricin as described previously (Novakova et al., 2002). Ethyl acetate extracts from the wild-type and the aurlR-disrupted strains were prepared from liquidgrown strains cultivated in the best auricin-production medium, Bennet medium, and analysed by TLC, followed by a bioassay against $B$. subtilis. As for many other antibiotics, production of auricin begins at the beginning of the stationary phase $(12 \mathrm{~h})$. No auricin was detected during exponential phase $(9 \mathrm{~h})$ in both strains (data not shown). At 




(b)



$\begin{array}{llll}12 & 14 & 16 & 18\end{array}$
Daur1R

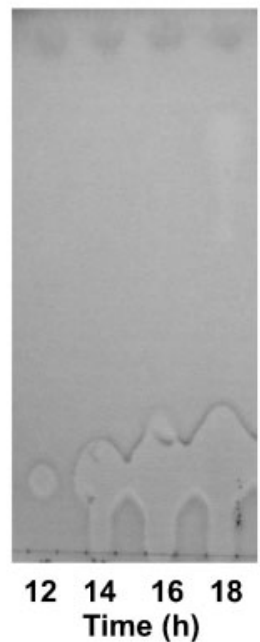

$\triangle$ aur1R + pPM-aur1R

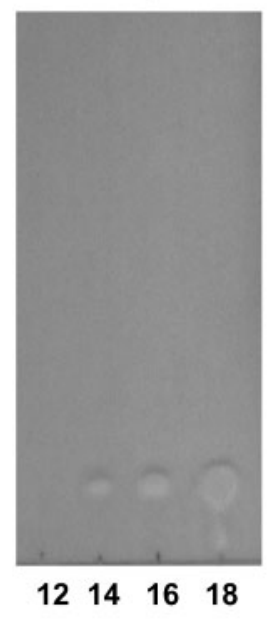

Fig. 1. (a) Growth curve of S. aureofaciens CCM 3239 wild-type (WT) and S. aureofaciens aur1R-disrupted $(\Delta$ aur1R) strains in liquid Bennet medium. Each point represents the mean of three assays; error bars, SD. (b) Analysis of auricin production in the wild-type, aur1R-disrupted and complemented $S$. aureofaciens strains by biochromatography assay. Ethyl acetate extracts from $S$. aureofaciens CCM 3239 wild-type (WT), aur1R-disrupted $(\triangle$ aur1R) and complemented ( $\triangle$ aur $1 R+\mathrm{pPM}-$ aur1R) strains grown to the indicated time points in Bennet medium were resolved by TLC and overlaid with $B$. subtilis, as described in Methods. The inhibition zone corresponds to auricin (Novakova et al., 2002). all time points, the inhibition zones corresponding to auricin in the aur1R-deletion mutant were clearly and reproducibly larger than those of the wild-type $S$. aureofaciens CCM 3239 strain (Fig. 1b). This inhibition zone was absent in the ethyl acetate extracts prepared either from the S. aureofaciens, aur1::tsr strain with deleted critical PKS genes (Novakova et al., 2002) or from the S. aureofaciens, aur1P:: tsr strain with a deleted auricin pathway-specific activator (Novakova et al., 2005), which were grown under identical conditions (results not shown). Therefore, it was obvious that this inhibition zone corresponded to auricin.

To verify that this phenotype was solely due to the deletion of aur1R, S. aureofaciens $\Delta a u r 1 R 1$ was complemented in trans by transformation with plasmid pPM-aur1R, containing the aur $1 R$ gene including its promoter cloned in the integrative plasmid pPM927. The level of auricin decreased in the complemented strain to a level even lower than in the wild-type strain (Fig. 1b), showing that the increase of auricin in $S$. aureofaciens $\Delta a u r 1 R 1$ was indeed due to the aur $1 R$ disruption. These results therefore indicated that the aur $1 R$ gene likely encodes a negative regulator of auricin production.

\section{Transcriptional analysis of aur1R}

Expression of aur1R was investigated by $\mathrm{S} 1$ nuclease mapping using probe 1 and RNA isolated from $S$. aureofaciens CCM 3239 at various stages of growth in liquid Bennet medium. As shown in Fig. 2(a), a single RNA-protected fragment was identified that corresponded to the aurlRp promoter with a TSP at A, 24 nucleotides upstream of the most likely translation initiation codon GTG (Fig. 4b). No RNA-protected fragment was identified with tRNA as a control (Fig. 2a, lane C). The promoter was regulated in a growth stage-dependent manner in Bennet medium and induced at the beginning of stationary phase, correlating with auricin production. The sequence of the aurlRp promoter and flanking regions is shown in Fig. 4 (b). The putative -10 region of aur1Rp shows quite high similarity (five out of six matches) to the -10 region of the consensus sequence recognized by the major Streptomyces sigma factor $\sigma^{\mathrm{HrdB}}$ (Brown et al., 1992), although the sequence around the -35 region shows only limited similarity (three out of six matches). The two regions are separated by an optimal spacer of $17 \mathrm{bp}$. Therefore, as for the divergent aur1Pp promoter (Novakova et al., 2005), it 
(a) aur1Rp

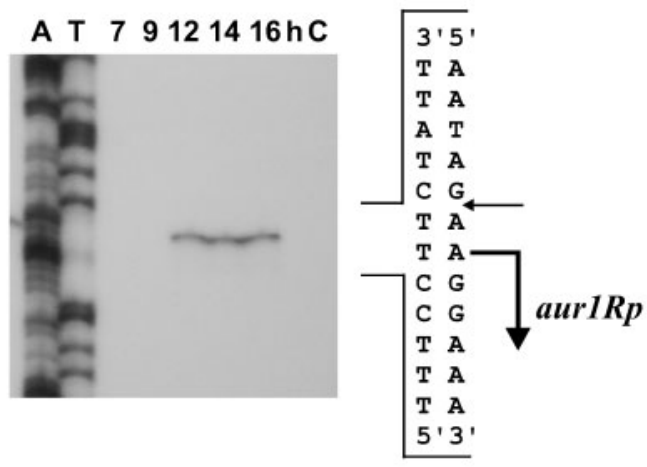

(b)
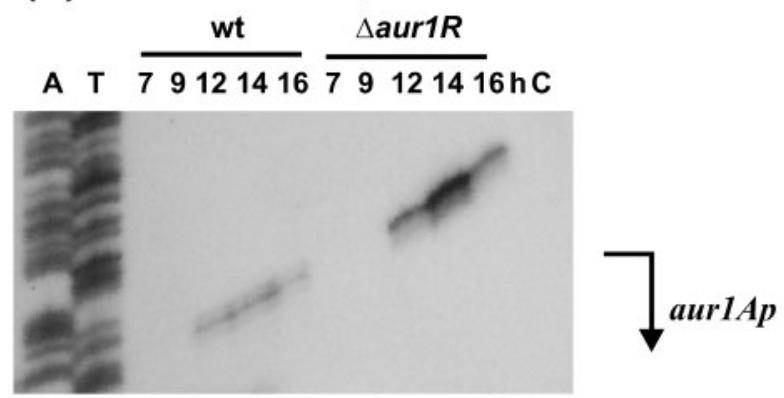

(c)



A T $\frac{w t}{79121416} \frac{\Delta \text { aur1R }}{79121416 \mathrm{hC}}$

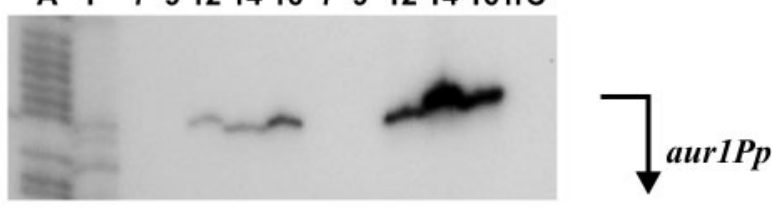

(d) hrdBp2
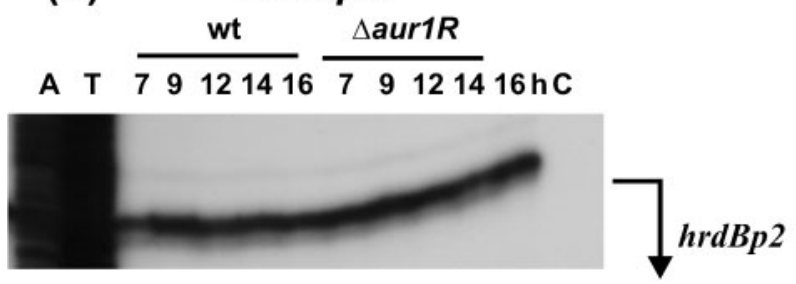

is likely that the aur $1 R p$ promoter is transcribed by an RNA polymerase holoenzyme containing the principal sigma factor $\sigma^{\mathrm{HrdB}}$.

\section{Dependence of aur1A and aur1P transcription on aur1R}

Previously, we had characterized the aurlAp promoter, which directs expression of the first characterized biosynthetic gene of the auricin cluster, aurlA, which encodes a
Fig. 2. High-resolution $S 1$ nuclease mapping of the TSP for the aur1Rp promoter in $S$. aureofaciens CCM 3239 wild-type strain (a), and for the aur1Pp and aur1Pp promoters in S. aureofaciens CCM 3239 wild-type (wt) and $S$. aureofaciens aur1R-disrupted $(\triangle \operatorname{aur} 1 R)$ strains $(\mathrm{b}, \mathrm{c})$. The 5 '-labelled DNA fragments were hybridized with $40 \mu \mathrm{g}$ RNA and treated with $120 \mathrm{U}$ S1 nuclease. RNA was isolated from the cultures grown in liquid Bennet medium at the time points indicated, which corresponded to the different growth phases (Fig. 1a): the exponential phase (7 and 9 h), the end of exponential phase $(12 \mathrm{~h})$ and the stationary phase (14 and 16 h). E. coli tRNA was used as a control (lane C). (d) Control S1 nuclease mapping experiments with the same RNA samples and a DNA probe for the hrdBp2 promoter (Kormanec \& Farkasovsky, 1993). The RNA-protected DNA fragments were analysed on DNA sequencing gels together with $G+A$ (lane $A$ ) and $T+C$ (lane $T$ ) sequencing ladders derived from the end-labelled fragments (Maxam \& Gilbert, 1980). The thin horizontal arrow indicates the position of an RNA-protected fragment and thick bent vertical arrows indicate the nucleotide corresponding to the TSP. Before assigning the TSP, 1.5 nucleotides was subtracted from the length of the protected fragment to account for the difference in the $3^{\prime}$ ends resulting from $S 1$ nuclease digestion and the chemical sequencing reactions. In every experiment, the same RNA preparations were hybridized in parallel with all of the probes. All S1 nuclease mapping experiments were performed twice with independent sets of RNA with similar results.

putative oxygenase (Novakova et al., 2002). Transcription of the aurlAp promoter was directly activated by the auricin pathway-specific activator Aur1P (Novakova et al., 2005). Therefore, to investigate whether aur $1 R$ disruption has an effect on transcription of both aurlA and aur1P genes, $\mathrm{S} 1$ nuclease mapping was performed using probes 2 and 3 and RNA isolated from S. aureofaciens CCM 3239 wild-type and the aur $1 R$ mutant strains cultivated in liquid Bennet medium, as for analysis of aur $1 R$ transcription. As shown in Fig. 2(b, c), with the RNA isolated from the wildtype strain, single RNA-protected fragments were identified with TSPs that corresponded to the aurlAp and aurlPp promoters, as published previously (Novakova et al., 2005). As for aurlRp, both promoters were inactive in the exponential growth phase (7 and $9 \mathrm{~h}$ ) and induced at the beginning of the stationary phase $(12 \mathrm{~h})$, which correlates with auricin production. However, the levels of aurlAp and aur $1 P p$ mRNA from all the time points were reproducibly increased in the aur $1 R$ mutant. As an internal control, S1 nuclease mapping was performed with the same RNA samples using a probe fragment specific for the $S$. aureofaciens $h r d B p 2$ promoter, which is expressed fairly constantly during growth and differentiation (Kormanec \& Farkasovsky, 1993). RNA-protected fragments of similar intensities corresponding to the $h r d B p 2$ promoter were identified with all RNA samples (Fig. 2d). The results indicated that the aur $1 R$ mutation positively affects transcription from both promoters. Therefore, increased transcription from the aur $1 P p$ and the aur $1 A p$ promoters is responsible for the increase in auricin production in the aurlR mutant. The results are also consistent with the 
putative role of Aur1R as a negative repressor of auricin expression.

\section{The Aur1R protein binds specifically to the aur1Pp and aur1Rp promoters and its binding is affected by auricin or its intermediate(s)}

To investigate whether Aur1R directly regulates aurlAp and aurlPp transcription, the aurlR gene was overexpressed in the E. coli T7 RNA polymerase expression system, and the purified N-terminal His-tagged Aur1R was examined for its binding to the aurlAp and aurlPp promoter regions by EMSA. Two ${ }^{32} \mathrm{P}$-labelled DNA fragments were used for the binding studies: a $200 \mathrm{bp}$ fragment encompassing the aurlAp promoter (nucleotide positions -167 to +32 with respect to the TSP) has been described previously for binding of AurlP (Novakova et al., 2005); and a 406 bp fragment comprising the aur $1 R-$ aurlP intergenic region (nucleotide positions -291 to
+115 with respect to the TSP for the aur1Pp promoter). No binding was identified with the aurlAp promoter DNA fragment (data not shown). However, using the $406 \mathrm{bp}$ aur1R-aur1P intergenic DNA fragment, four retarded bands were clearly visible with an increasing concentration of Aur1R, which may correspond to four different complexes (Fig. 3). The specificity of the interaction was demonstrated by the competitive binding of the unlabelled fragment (Fig. 3, lane 6). These results indicated that Aur1R is capable of binding to the aurlR-aurlP intergenic region.

To investigate the possibility that auricin or its intermediate is an effector ligand for Aur1R, the culture supernatants of $S$. aureofaciens CCM 3239 and its isogenic S. aureofaciens, aur $1:$ : $t s$ mutant with a deletion of critical PKS genes (Novakova et al., 2002), grown for $14 \mathrm{~h}$ in Bennet medium, were extracted with ethyl acetate, dried and dissolved in methanol. Decreasing amounts of extracts were used in a series of twofold dilutions in binding

(a)



(b)

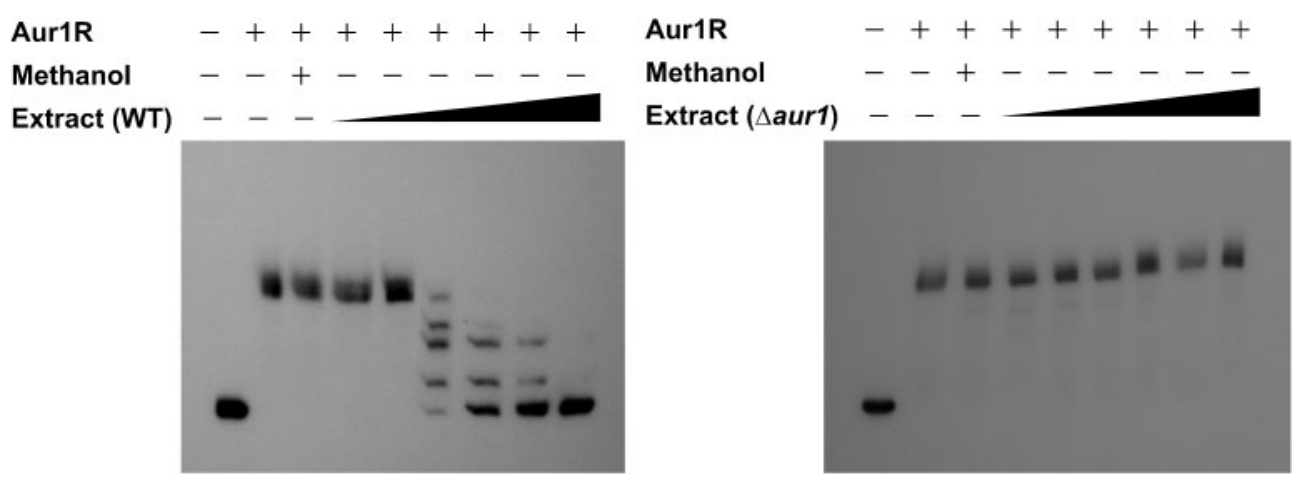

Fig. 3. (a) Detection of protein-DNA complexes by EMSA using 0.04 ng of a ${ }^{32} \mathrm{P}$-labelled 406 bp DNA fragment comprising the aur1R-aur1P intergenic region (probe 2) with increasing amounts of purified His-tagged Aur1R. Lane 1, labelled fragment in the absence of protein; lanes 2-5, 2, 5, 10 and $15 \mu \mathrm{g}$, respectively, of the purified His-tagged Aur1R protein. Addition of $200 \mathrm{ng}$ unlabelled $406 \mathrm{bp}$ aur1R-aur1P intergenic DNA fragment was used to demonstrate Aur1R binding specificity (lane 6). The arrows indicate the free DNA fragment and shifted fragments corresponding to the proposed complexes. (b) Effect of ligand(s) on the Aur1R binding activity. EMSA was done with $0.01 \mathrm{ng}{ }^{32} \mathrm{P}$-labelled probe 2 and $4 \mu \mathrm{g}$ Aur1R with increasing amounts of culture extracts from the wild-type (WT) and the S. aureofaciens aur1 : : tsr mutant ( $\Delta$ aur1; Novakova et al., 2002) in a series of twofold dilution samples. Methanol was used as a solvent. A $1 \mu$ lvolume of methanol or the particular extract was used in each $15 \mu \mathrm{l}$ binding reaction. A $1 \mu \mathrm{l}$ volume of the extract corresponded to the amount of auricin present in $1.67 \mathrm{ml}$ liquid culture prepared as described in Methods. 
(a)



\section{Non-coding strand}

A T 123

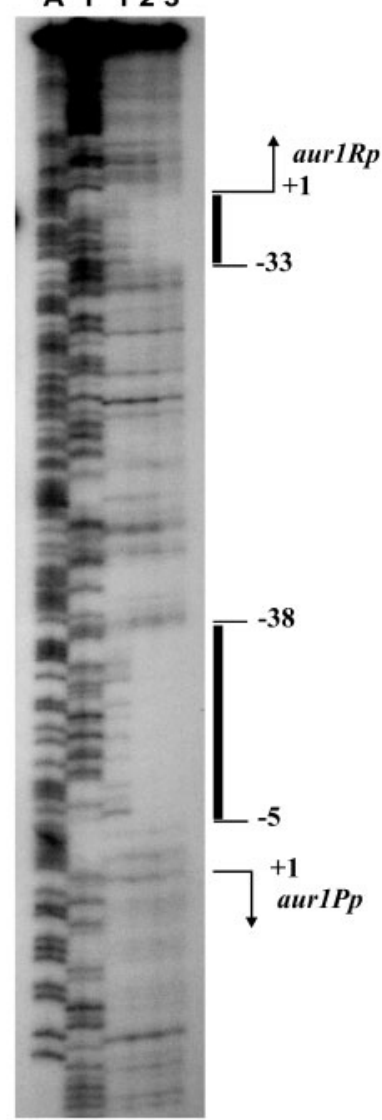

(b)

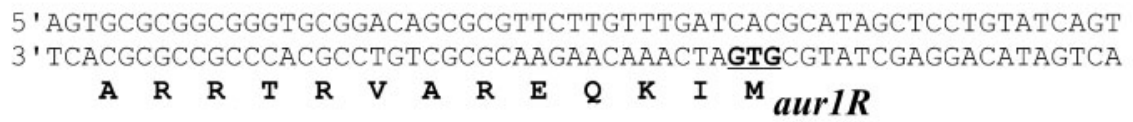

TTCCTTCTATTATTAAACCGTCCCGTTGGTATTGGAAGCAGGTGTGGGTCCCCCTGGATG AAGGAAATAATAATTTGGCAGGGCAACCATAACCTTOGTCCACACCCAGGGGGACCTAC

aurlRp -10

GCCTGTTCTGCGTGGTTATTCGCCTGTCGATGGCCCGATTTAACCCTTTTATCGGCAACA

CGGACAAGACGCACCAATAAGCGGACAGCTACCGGGCTAAATTGGGAAAATAGCCGTTGT

CGGCTCCGTTTTGTGGGCTTTGACAAACCGACGCAGCGGTTTGTATCTTCTTGA्ACGTTGA

GCCGAGGCAAAACACCCGAAACTGTTTGGCTGCGTCGCCAAACATAGAAGAACTGCAACT



CCCAACCGAGCATGAGGGAGGGGGGCAGATGAACCAGCGGACCATGGCTGTGCTGGAACC 3 '

GGGTTGGCTCGTACTCCCTCCCCCCGTCTACTTGGTCGCCTGGTACCGACACGACCTTGG 5 '

Fig. 4. (a) DNase I footprints of Aur $1 \mathrm{R}$ binding to $0.3 \mathrm{ng}$ of a $5^{\prime}$ end-labelled, either coding or non-coding strand, 406 bp DNA fragment encompassing the aur1R-aur1P intergenic region (probe 1 and probe 2, respectively). The vertical bars indicate the positions of the Aur1R binding sites. The numbering is relative to the TSP of the aur1Pp and au1Rp promoters. The TSPs of the promoters are indicated by bent arrows. Lane 1 is without the His-tagged Aur1R protein sample. Lanes 2 and 3 contain 30 and $75 \mu \mathrm{g}$, respectively, of the purified His-tagged Aur1R protein. Lanes $A$ and $T$ represent $G+A$ and $C+T$ sequencing ladders, respectively (Maxam \& Gilbert, 1980). All binding experiments were performed twice with independent sets of protein samples, giving similar results. (b) The nucleotide sequence of the $S$. aureofaciens CCM 3239 aur1R-aur1P intergenic region. The deduced protein products are given using the single-letter amino acid code at the second position of each codon. The TSPs of the aur1Pp and aur1Rp promoters are indicated by bent arrows. The putative -10 and -35 boxes of the promoters are in bold type and underlined. The nucleotides that were protected from DNase I by Aur1R binding are shaded. The nucleotide sequence shown is a part of the aur1 cluster that has been deposited in GenBank/EMBL/DDBJ databases under accession number AY956334. 
reactions of the $406 \mathrm{bp}$ aur1R-aur1P intergenic DNA fragment with a constant concentration of Aur1R. An increasing concentration of extract from the wild-type $S$. aureofaciens dissociated Aur1R from the promoter regions, but similar amounts of extract from the aurl-disrupted $S$. aureofaciens strain had no effect on Aur1R binding (Fig. 3b).

To map the binding sites for Aur1R within the region, a DNase I footprinting assay was carried out using the same $406 \mathrm{bp}$ aur $1 R$-aur1P intergenic DNA fragment, 5' endlabelled at either end. Using both coding and non-coding strands, two clearly separated regions were protected. Interestingly, both protected regions covered the aurlRp and aurlPp promoters, respectively, in almost identical positions with respect to the TSPs for the promoters, occupying both putative -10 and -35 promoter elements (Fig. 4). This repressor mode of binding to the promoters is consistent with the proposed function of AurlR as a negative transcriptional regulator belonging to the TetR family. Inspection of both DNase I-protected regions in the aurlRp and aurlPp promoters revealed that both binding regions are similar and contain a partial inverted repeat that is partially similar to the autoregulatory element (ARE) consensus sequence of the Streptomyces $\gamma$-butyrolactone autoregulator receptor proteins (Fig. 5) (Folcher et al., 2001). The results of the binding studies indicated that the effect of aurlR upon aurlP expression is direct, and that the effect of aurlR upon aur $1 A$ expression is likely indirect, through the activation of Aur1P, which has been shown to directly bind to the aurlAp promoter (Novakova et al., 2005). The results suggested a cascade regulation of the aurl cluster via negative and positive modes. In addition to aurlPp, Aur1R also bound to its own aurlRp promoter, indicating negative feedback control.

\section{DISCUSSION}

Antibiotic biosynthesis is very often tightly regulated at several levels to ensure exact timing of production and to avoid the potential toxicity of an antibiotic to the producer. The activity of the pathway-specific regulatory genes is often regulated by genes at a higher level, including global regulatory genes mediating environmental, nutritional and growth-rate effects (Bibb, 2005). The results of the present study indicate a similar regulatory cascade for the angucycline-like auricin gene cluster in $S$. aureofaciens CCM 3239. As for other similar angucycline gene clusters, auricin is produced with very low yields that indicate tight regulation. Two regulatory genes, aur $1 P$ and aurlR, have been identified in the auricin polyketide gene cluster. The aur $1 P$ gene, encoding a homologue of response regulators, has been shown to represent a pathway-specific transcriptional activator that directly controls the activity of the aurlAp promoter, which governs transcription of the first biosynthetic gene, aur $1 A$, in the auricin gene cluster in S. aureofaciens CCM 3239 (Novakova et al., 2005). The present results demonstrate that the divergently located aur $1 R$ gene has a negative role in regulation of auricin production through a direct inhibition of aurlP expression. This repressor mode of Aur1R binding to the aurlPp promoter is likely relieved by an interaction of Aur1R with auricin or its intermediate(s). Moreover, Aur1R also bound its own promoter in an almost identical way, which indicates a negative feedback autoregulatory control of aurlR expression.

The aur $1 R$ gene encodes a protein homologous to members of the TetR family of transcriptional regulators, which function mainly as repressors. Like many other TetRfamily repressors, Aur1R consists of an N-terminal conserved HTH DNA-binding domain and a variable Cterminal effector-binding domain. (Ramos et al., 2005). The highest similarity of the Aur1R DNA-binding domain was to members of the subfamily of $\gamma$-butyrolactone autoregulator pseudo-receptors, e.g. JadR2 (Yang et al., 1995), TylQ (Stratigopoulos \& Cundliffe, 2002), BarB (Matsuno et al., 2004) and AlpW (Bunet et al., 2008), which all constitute an independent branch in the phylogenetic tree of $\gamma$-butyrolactone autoregulator receptors and likely do not bind $\gamma$-butyrolactone autoregulators (Nishida et al., 2007). Like Aur1R, almost all the pseudoreceptors act as negative regulators of antibiotic production and play a role in the specific repression of pathwayspecific transcription activator genes of the particular secondary metabolite gene cluster. Many of them have also been shown to be under the direct control of bona fide



Fig. 5. Comparison of the nucleotide sequences of the aur1Pp and aur1Rp promoters protected by Aur1R with the autoregulatory element (ARE) consensus sequence of the Streptomyces $\gamma$-butyrolactone autoregulator receptor proteins (Folcher et al., 2001). Identical residues are highlighted in black. Arrows indicate the position of the $22 \mathrm{bp}$ inverted repeat sequence identified as a consensus sequence (ARE box) of the $\gamma$ butyrolactone autoregulator receptor protein ArpA (Onaka \& Horinouchi, 1997). 
$\gamma$-butyrolactone autoregulator receptors (Matsuno et al., 2004; Stratigopoulos et al., 2002). Therefore, it seems likely that pseudo-receptor genes play a common role in the negative regulation of secondary metabolite biosynthesis as target genes of autoregulator receptors in the autoregulator signalling cascade. Our preliminary experiments revealed a likely true $\gamma$-butyrolactone autoregulator receptor which bound the aurlRp promoter in S. aureofaciens CCM 3239, thus indicating a similar regulation of auricin biosynthesis (J. Kormanec, unpublished results).

The members of the TetR family are controlled by small effector ligands, which after interaction with the protein inhibit their binding to the cognate operator sites (Ramos et al., 2005). Several Streptomyces antibiotic pathway TetR family regulators (DnrO, SimR, LanK and ActR) have been shown to respond to the mature antibiotic and/or pathway intermediates as effector ligand (Jiang \& Hutchinson, 2006; Le et al., 2009; Ostash et al., 2008; Tahlan et al., 2007). However, no effector ligand has yet been identified for this subfamily of $\gamma$-butyrolactone autoregulator pseudoreceptors. Aur1R is the first example of a member of this subfamily regulated by an antibiotic or its intermediate(s).

An interesting feature of AurlR is its manner of binding to aur1Rp and aur1Pp promoters. While DNase I footprinting analysis revealed two binding regions that overlapped both promoters (Fig. 4), four different complexes have been identified by EMSA using a DNA fragment encompassing both promoters with increasing amounts of Aur1R (Fig. 3). One explanation might be that one molecule of Aur1R binds one half of the palindromic operator site (Fig. 5). Assuming different AurlR affinities to particular parts of the operators, we could explain four different complexes with increasing Aur1R concentration in EMSA analysis. However, this scenario is rather unlikely, as members of the TetR family in general bind their recognition sequences as dimers (Ramos et al., 2005). The other possibility is that two AurlR dimers bind a single operator comprising a 22 bp inverted repeat (Fig. 5) cooperatively, as has been described for another TetR homologue, QacR. In contrast to TetR, which binds a $15 \mathrm{bp}$ inverted repeat operator as a single dimer, two dimers of QacR bind a longer operator site (a single 28 bp inverted repeat) cooperatively from the opposite sites of the DNA helix (Schumacher et al., 2002). The longer Aur1R operator inverted repeat (22 bp) might indicate this mechanism. However, a detailed structural analysis would be needed to explain more clearly how AurlR binds to its operator site.

Based on the present data we can conclude that Aur $1 \mathrm{R}$ is a negative regulator of auricin biosynthesis through inhibition of transcription of the gene encoding the pathwayspecific activator Aur1P. We suggest that the role of Aur1R is to repress transcription of aur1P until the bacterial cells are fully prepared for auricin production, by for example permitting earlier expression of auricin resistance. Auricin or its intermediates likely represent ligand(s) that after binding to Aur1R relieve its binding to the aurlPp promoter and consequently induce its transcription, resulting in accumulation of threshold amounts of the pathway-specific activator Aur1P to induce expression of auricin biosynthetic genes. Recent results concerning an Aur1P homologue, JadR1, confirm that jadomycin and its intermediate bind to JadR1 and that this binding constitutes a feedback control of jadomycin biosynthesis in Streptomyces venezuelae (Wang et al., 2009). It is likely that a similar feedback mechanism also exists for the auricin cluster. The results of the transcriptional analysis of the aur $1 A$ and aur $1 P$ genes (Fig. 2), where the level of both transcripts decreased in the later stages, are consistent with this hypothesis. Therefore, after successful purification and structural analysis of auricin, similar experiments to investigate auricin activation will be pursued.

\section{ACKNOWLEDGEMENTS}

We are grateful to Dr Mark Buttner (John Innes Centre, Norwich, UK) for plasmid pPM929 and to Dr Bertold Gust (John Innes Centre, Norwich, UK) for kindly providing all the plasmids and strains used in the PCR-targeting system; the system was supplied by Plant Bioscience Ltd (Norwich, UK). We thank Paul Dyson for critical reading of the manuscript. This work was supported by the Slovak Research and Development Agency under contract no. APVV-001707 and a VEGA grant 2/0104/09 from the Slovak Academy of Sciences.

\section{REFERENCES}

Ausubel, F. M., Brent, R., Kingston, R. E., Moore, D. D., Seidman, J. G., Smith, J. A. \& Struhl, K. (1995). Current Protocols in Molecular Biology. New York: Wiley.

Bibb, M. J. (2005). Regulation of secondary metabolism in streptomycetes. Curr Opin Microbiol 8, 208-215.

Bradford, M. M. (1976). A rapid and sensitive method for the quantitation of microgram quantities of protein utilizing the principle of protein-dye binding. Anal Biochem 72, 248-254.

Brown, K. L., Wood, S. \& Buttner, M. J. (1992). Isolation and characterization of the major vegetative RNA polymerase of Streptomyces coelicolor A3(2); renaturation of a sigma subunit using GroEL. Mol Microbiol 6, 1133-1139.

Bunet, R., Mendes, M. V., Rouhier, N., Pang, X., Hotel, L., Leblond, P. \& Aigle, B. (2008). Regulation of the synthesis of the angucyclinone antibiotic alpmycin in Streptomyces ambofaciens by the autoregulator receptor AlpZ and its specific ligand. J Bacteriol 190, 3293-3305.

Evans, G. A., Lewis, K. \& Rothenberg, B. E. (1989). High efficiency vectors for cosmid microcloning and genomic analysis. Gene 79, 9-20.

Folcher, M., Gaillard, H., Nguyen, L. T., Nguyen, K. T., Lacroix, P., Bamas-Jacques, N., Rinkel, M. \& Thompson, C. J. (2001). Pleiotropic functions of a Streptomyces pristinaespiralis autoregulator receptor in development, antibiotic biosynthesis, and expression of a superoxide dismutase. J Biol Chem 276, 44297-44306.

Gust, B., Challis, G. L., Fowler, K., Kieser, T. \& Chater, K. F. (2003). PCR-targeted Streptomyces gene replacement identifies a protein domain needed for biosynthesis of the sesquiterpene soil odor geosmin. Proc Natl Acad Sci U S A 100, 1541-1548.

Horinouchi, S., Hara, O. \& Beppu, T. (1983). Cloning of a pleiotropic gene that positively controls biosynthesis of A-factor, actinorhodin, 
and prodigiosin in Streptomyces coelicolor A3(2) and Streptomyces lividans. J Bacteriol 155, 1238-1248.

Jiang, H. \& Hutchinson, C. R. (2006). Feedback regulation of doxorubicin biosynthesis in Streptomyces peucetius. Res Microbiol 157, 666-674.

Kieser, T., Bibb, M. J., Buttner, M. J., Chater, K. F. \& Hopwood, D. A. (2000). Practical Streptomyces Genetics. Norwich, UK: The John Innes Foundation.

Kormanec, J. (2001). Analyzing the developmental expression of sigma factors with S1-nuclease mapping. In Nuclease Methods and Protocols. Methods in Molecular Biology, vol. 160, pp. 481-494. Edited by C. H. Chein. Totowa, NJ: Humana Press.

Kormanec, J. \& Farkasovsky, M. (1993). Differential expression of principal sigma factor homologues of Streptomyces aureofaciens correlates with developmental stage. Nucleic Acids Res 21, 3647-3652.

Kormanec, J., Rezuchova, B. \& Farkasovsky, M. (1993). Optimization of Streptomyces aureofaciens transformation and disruption of the $h r d A$ gene encoding a homologue of the principal $\sigma$ factor. J Gen Microbiol 139, 2525-2529.

Laemmli, U. K. (1970). Cleavage of structural proteins during assembly of the head of bacteriophage T4. Nature 227, 680-685.

Le, T. B., Fiedler, H.-P., den Hengst, C. D., Ahn, S. K., Maxwell, A. \& Buttner, M. J. (2009). Coupling of the biosynthesis and export of the DNA gyrase inhibitor simocyclinone in Streptomyces antibioticus. Mol Microbiol 72, 1462-1474.

Lombo, F., Brana, A. F., Salas, J. A. \& Mendez, C. (2004). Genetic organization of the biosynthetic gene cluster for the antitumor angucycline oviedomycin in Streptomyces antibioticus ATCC 11891. ChemBioChem 5, 1181-1187.

Matsuno, K., Yamada, Y., Lee, C.-K. \& Nihira, T. (2004). Identification by gene deletion analysis of barB as a negative regulator controlling an early process of virginiamycin biosynthesis in Streptomyces virginiae. Arch Microbiol 181, 52-59.

Maxam, A. M. \& Gilbert, W. (1980). Sequencing end-labeled DNA with base specific chemical cleavages. Methods Enzymol 65, 499-560.

Metsa-Ketela, M., Ylihonko, K. \& Mantsala, P. (2004). Partial activation of a silent angucycline-type gene cluster from a rubromycin b producing Streptomyces sp. PGA64. J Antibiot 57, 502-510.

Nishida, H., Ohnishi, Y., Beppu, T. \& Horinouchi, S. (2007). Evolution of $\gamma$-butyrolactone synthases and receptors in Streptomyces. Environ Microbiol 9, 1986-1994.

Novakova, R., Bistakova, J., Homerova, D., Rezuchova, B. \& Kormanec, J. (2002). Cloning and characterization of a polyketide synthase gene cluster involved in biosynthesis of a proposed angucycline-like polyketide auricin in Streptomyces aureofaciens CCM 3239. Gene 297, 197-208.

Novakova, R., Homerova, D., Feckova, L. \& Kormanec, J. (2005). Characterization of a regulatory gene essential for the production of the angucycline-like polyketide antibiotic auricin in Streptomyces aureofaciens CCM 3239. Microbiology 151, 2693-2706.

Onaka, H. \& Horinouchi, S. (1997). DNA-binding activity of the Afactor receptor protein and its recognition DNA sequence. Mol Microbiol 24, 991-1000.

Ostash, I., Ostash, B., Luzhetskyy, A., Bechthold, A., Walker, S. \& Fedorenko, V. (2008). Coordination of export and glycosylation of landomycins in Streptomyces cyanogenus S136. FEMS Microbiol Lett 285, 195-202.

Pang, X., Aigle, B., Girardet, J.-M., Mangenot, S., Pernodet, J.-L., Decaris, B. \& Leblond, P. (2004). Functional angucycline-like antibiotic gene cluster in the terminal inverted repeats of the Streptomyces ambofaciens linear chromosome. Antimicrob Agents Chemother 48, 575-588.

Ramos, J. L., Martinez-Bueno, M., Molina-Henares, A. J., Teran, W., Watanabe, K., Zhang, X., Galledos, M. T., Brennan, R. \& Tobes, R. (2005). The TetR family of transcriptional repressors. Microbiol Mol Biol Rev 69, 326-356.

Redenbach, M., Kieser, H. M., Denapaite, D., Eichner, A., Cullum, J., Kinashi, H. \& Hopwood, D. A. (1996). A set of ordered cosmids and a detailed genetic and physical map for the $8 \mathrm{Mb}$ Streptomyces coelicolor A3(2) chromosome. Mol Microbiol 21, 77-96.

Schumacher, M. A., Miller, M. C., Grkovic, S., Brown, M. H., Skurray, R. \& Brennan, R. G. (2002). Structural basis for cooperative DNA binding by two dimers of the multidrug-binding protein QacR. EMBO J 21, 1210-1218.

Smokvina, T., Mazodier, P., Boccard, F., Thompson, C. J. \& Guerineau, M. (1990). Construction of a series of pSAM2-based integrative vectors for use in actinomycetes. Gene 94, 53-59.

Stratigopoulos, G. \& Cundliffe, E. (2002). Expression analysis of the tylosin-biosynthetic gene cluster: pivotal regulatory role of the tylQ product. Chem Biol 9, 71-78.

Stratigopoulos, G., Gandecha, A. R. \& Cundliffe, E. (2002). Regulation of tylosin production and morphological differentiation in Streptomyces fradiae by TylP, a deduced $\gamma$-butyrolactone receptor. Mol Microbiol 45, 735-744.

Tahlan, K., Ahn, S. K., Sing, A., Bodnaruk, T. D., Willems, A. R., Davidson, A. R. \& Nodwell, J. R. (2007). Initiation of actinorhodin export in Streptomyces coelicolor. Mol Microbiol 63, 951-961.

Wang, L., Tian, X., Wang, J., Yang, H., Fan, K., Xu, G., Yang, K. \& Tan, H. (2009). Autoregulation of antibiotic biosynthesis by binding of the end product to an atypical response regulator. Proc Natl Acad Sci U S A 106, 8617-8622.

Yang, K., Han, L. \& Vining, L. C. (1995). Regulation of jadomycin B production in Streptomyces venezuelae ISP5230: involvement of a repressor gene, jadR2. J Bacteriol 177, 6111-6117.

Edited by: L. Heide 